\title{
Identification of Candida spp. isolated from oral mucosa in patients with leukemias and lymphomas in Iran
}

\author{
Sara Hamzehee' ${ }^{1}$, Davood Kalantar-Neyestanaki², Mohammad Ali Mohammadi², \\ Saeed Nasibi ${ }^{3}$, Seyed Amin Ayatollahi Mousavi ${ }^{*}$
}

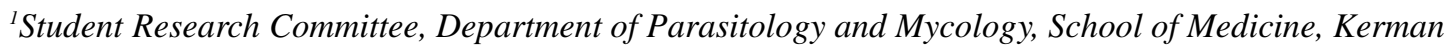 \\ University of Medical Sciences, Kerman, Iran \\ ${ }^{2}$ Department of Microbiology and Virology, School of Medicine, Kerman University of Medical Sciences, \\ Kerman, Iran \\ ${ }^{3}$ Research Center for Hydatid Disease in Iran, Kerman University of Medical Sciences, Kerman, Iran \\ ${ }^{4}$ Department of Medical Mycology and Parasitology, School of Medicine, Kerman University of Medical \\ Sciences, Kerman, Iran
}

Received: December 2018, Accepted: March 2019

\begin{abstract}
Background and Objectives: Oral candidiasis is a serious problem for immunocompromised patients, especially patients with hematological malignancies. After becoming a systemic candidiasis it is difficult to diagnose, control and treat in individuals with hematological malignancies. The aim of this study was to diagnose candidiasis in the oral mucosa of patients with leukemias and lymphomas in a timely manner in order to prevent their progression to systemic candidiasis.

Materials and Methods: In this cross sectional study, 50 clinical samples were collected from the mouth of patients with hematological malignancies undergoing chemotherapy from the oncology units of teaching hospitals in Kerman, Iran. Patients were from Kerman, Sistan-Baluchestan and Hormozgan in south-eastern Iran. Sampling was restricted to patients with diagnosed acute lymphoid leukemia (ALL); acute myeloid leukemia (AML); chronic lymphoid leukemia (CLL); chronic myeloid leukemia (CML); Hodgkin's lymphoma (HL) and non-Hodgkin's lymphoma (NHL). Presumptive species identification of fungi was performed using conventional methods like colony characteristics on CHROMagar Candida medium, germ tube production, and assessing the morphology fungi on corn meal agar. Confirmation of presumptive candida isolates was performed using PCR-RFLP.

Results: From a total of 50, 14 patients (28\%) had positive oral candidiasis. Candida albicans (57.14\%) was the most common species followed by Candida glabrata (14.28\%), Candida parapsilosis (14.28\%), Candida krusei (7.14\%) and Candida kefyr (7.14\%). Candida albicans had the highest rate of oral infection in ALL (35.71\%) and then NHL (28.57\%) patients. Conclusion: The results indicate that oral candidiasis is a prevalent fungal infection in the patients with hematologic malignancies with $C$. albicans being the main etiological agent. However, other species of Candida cause similar infections in these patients.
\end{abstract}

Keywords: Candida spp.; Oral candidiasis; Hematologic malignancies; Lymphomas

${ }^{*}$ Corresponding author: Seyed Amin Ayatollahi Mousavi, PhD, Department of Medical Mycology and Parasitology, School of Medicine, Kerman University of Medical Scienc- es, Kerman, Iran.

Email: aminayatollahi@kmu.ac.ir 


\section{INTRODUCTION}

Candida species are opportunistic fungi. The most cases of candidiasis are caused by $C$. albicans followed by $C$. glabrata, C. parapsilosis, C. tropicalis, C. krusei and C. kefyr (1). Oral candidiasis is one of the most important forms of mucosal candidiasis. A weakened mucosal barrier permits translocation of fungi present in the mouth, oropharynx, gastrointestinal tract or skin to enter the body and, when this is combined with immunosuppression, severe infections are common (2). The incidence of opportunistic fungal infections such as oral candidiasis has increased in the past decades (3). Oral candidiasis represents a serious problem for patients with hematologic malignancies (4). There are many risk factors that make patients susceptible to candidiasis. These risk factors include: a prolonged hospital stay, blood malignancy, treatment with broad-spectrum antibiotics, neoplastic diseases, and repressive treatment of the immune system for organ transplants, intubation and catheter use. Based on the increase in number of patients with such risk factors, incidence of candidiasis also has increased (5). Often these infections happen in patients with hematologic malignancies. This increase depends on the host defense system in the following circumstances: changes in chemotherapy, hematopoietic stem cells transplantation, and abstract radiation use of corticosteroids, cyclosporine and other immunosuppressive agents (6-11). Cancer patients are exposed to localized and diffuse infections, such as oral infections due to illness and chemotherapy-related symptoms, with weakening of the immune system. Cytotoxic chemotherapy, radiation or malignancies themselves are known to compromise the cell-mediated host immunity which plays a significant function in the control of fungal infections. Recent studies express that there is a variation in the incidence of oral yeast colonization and infection amongst different cancer groups (12).

In this study, molecular method, PCR-RFLP, was used to detect candidiasis in the oral mucosa of patients with leukemia and lymphoma in a timely manner in order to prevent their progression to systemic candidiasis. After becoming a systemic candidiasis, it is difficult to diagnose, control the infection in patients with hematological malignancies, especially those with leukemia and lymphoma, and in many cases, this can lead to increase mortality (13).

\section{MATERIALS AND METHODS}

Patients and specimens collection. Fifty swabs were collected from the mouths of patients with hematologic malignancies undergoing chemotherapy from the oncology units of the teaching hospitals (Afzalipour, Shahid-Bahonar and Shafa Hospitals) in Kerman, Iran, from March 2017 to February 2018. Patients were from Kerman, Sistan-Baluchestan and Hormozgan provinces of Iran. Fifty samples including 13 patients with acute lymphoid leukemia (ALL); 13 patients, acute myeloid leukemia (AML); 13 patients, chronic lymphoid leukemia (CLL); 5 patients, chronic myeloid leukemia (CML); 5 patients, Hodgkin's lymphoma (HL); 4 patients and non-Hodgkin's lymphoma (NHL); 10 patients.

Samples were collected and transferred to the medical mycology laboratory of Kerman University of Medical Sciences. Mouth swabs were subjected to direct examination with $20 \% \mathrm{KOH}$ and cultured on Sabouraud's dextrose agar (Merck, Germany) containing chloramphenicol $(0.5 \mu \mathrm{g} / \mathrm{mL})$ (Merck KGa A, Darmstadt, Germany). All isolates were presumptively identified by phenotypic methods such as the color of colonies on CHROMagar Candida medium (CHROMagar, India, Cat no: 212961), germ-tube formation in serum and production of chlamydoconidia in corn meal agar (Merck, Germany) with $1 \%$ Tween 80 .

Molecular identification: DNA extraction and PCR-RFLP. The genomic DNA was extracted from yeast cultures using the Exgene cell SV-mini $10^{6}-10^{1}$ DNA tissue kit (GeneAll, South Korea). PCR amplification was performed using universal primers ITS1 (5'-TCCGTAGGTGAACCTGCGG-3') and ITS4 (5'-TCCTCCGCTTATTGATATGC-3') in a thermal cycler instrument (Biometra GmbH, Germany) (14). The PCR reaction contained $1 \mu \mathrm{L}$ of DNA, $10 \mu \mathrm{L}$ of master mix $2 \times$ (YTA 1553, Iran), $1 \mu \mathrm{L}$ of each primers, and $12 \mu \mathrm{L}$ of distilled water. The cycling program was: an initial denaturation at $94^{\circ} \mathrm{C}$ for 5 min followed by 30 cycles of $94^{\circ} \mathrm{C}$ for $60 \mathrm{~s}, 60^{\circ} \mathrm{C}$ for $60 \mathrm{~s}, 72^{\circ} \mathrm{C}$ for $120 \mathrm{~s}$ and final extension at $72^{\circ} \mathrm{C}$ for $10 \mathrm{~min}$. The PCR products were electrophoresed in $1 \%$ agarose gel in $0.5 \times$ TBE buffer and stained with ethidium bromide and then visualized by a Gel Doc system.

Restriction fragment length polymorphism 
analysis. MspI (HpaII) (Thermo scientific, USA) restriction enzyme was used for RFLP (Table 1). Digestion with MSPl enzyme performed by incubating $10 \mu \mathrm{L}$ of PCR reaction mixture, $18 \mu \mathrm{L}$ nuclease- free water, $2 \mu \mathrm{L} 10 \times$ buffer Tango and $1 \mu \mathrm{L}$ of each enzymes in a final reaction volume of $31 \mu \mathrm{L}$ at $37^{\circ} \mathrm{C}$ for $3 \mathrm{~h}$. Restriction fragments were separated in $2 \%$ agarose gel by electrophoresis in $0.5 \times$ TBE buffer, then visualized by a Gel Doc system after staining the with ethidium bromide.

\section{RESULTS}

A total of 50 patients participated in this study, with 14 of them $(28 \%)$ had positive oral candidiasis.

The species identification was acheived using conventional methods such as growth characteristics on Sabouraud's dextrose agar, color of colony on CHROMagar Candida medium, germ tube production and assessing the morphology on corn meal agar. All isolates were re-identified by PCR-RFLP method (Figs. 1, 2).

C. albicans $(\mathrm{n}=8,57.14 \%)$ was the most common species among them followed by $C$. glabrata $(\mathrm{n}=2$, $14.28 \%)$, C. parapsilosis $(\mathrm{n}=2,14.28 \%)$, C. krusei $(\mathrm{n}=1,7.14 \%)$ and $C$. kefyr $(\mathrm{n}=1,7.14 \%)$. The distribution of Candida species isolated from oral candidiasis in patients with hematologic malignancies undergoing chemotherapy, according to clinical presentation and type of cancer, are shown in Fig. 3. The distribution of Candida species according to type of hematologic malignancies presented in Table 2. C. albicans was the most frequent species isolated in the hematologic malignant disease.

Table 1. DNA size for PCR products (bp) of Candida spp., after RFLP.

\begin{tabular}{lll}
\hline Candida spp & ITS $_{\mathbf{1}}$ ITS $_{\mathbf{4}}$ & MSP $_{\mathbf{1}}$ \\
\hline C. albicans & 535 & $238-297$ \\
C. Parapsilosis & 520 & 520 \\
C. Tropicalis & 524 & $184-340$ \\
C. krusei & 510 & $249-261$ \\
C. guilliermondii & 608 & $82-155-371$ \\
C. glabrata & 871 & $314-577$ \\
C. lusitaniae & 383 & $117-266$ \\
C. kefyr & 721 & 721 \\
\hline
\end{tabular}

\section{DISCUSSION}

The incidence of opportunistic fungal infections such as oral candidiasis has increased in the past few

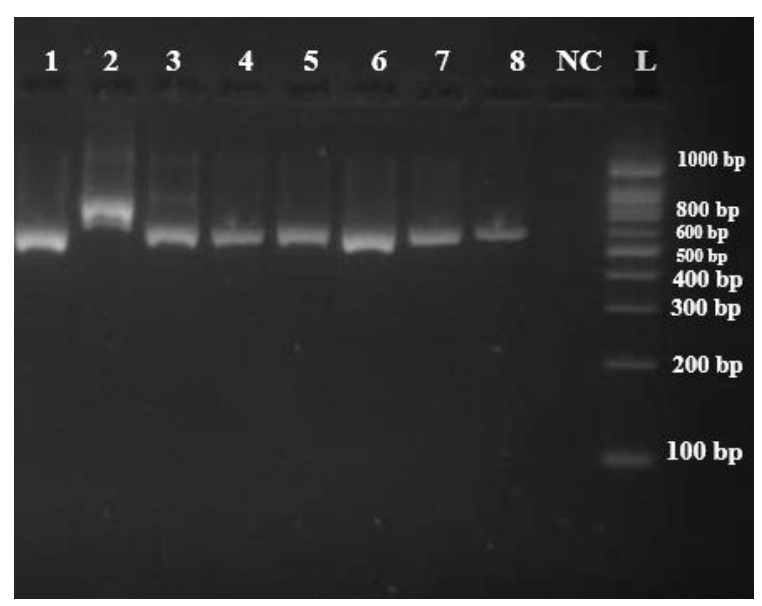

Fig. 1. Isolates 1 and 8: Amplification of genomic DNA from clinical isolates using ITS1 and ITS4 primers, NC: Negative Control, L: 100 bp DNA size marker.

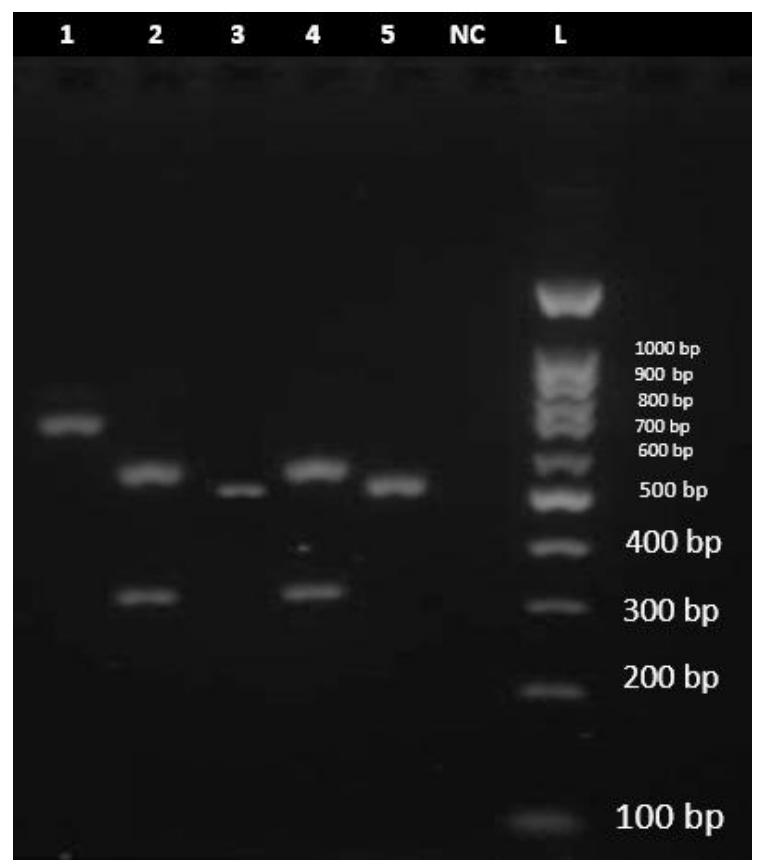

Fig. 2. Agarose gel electrophoresis of ITS-PCR products of various pathogenic Candida species after digestion with MspI. Lanes 1-5: C. kefyr (721 bp), C. glabrata (314 bp, 577 bp), C. parapsilosis (520 bp), C. glabrata (314 bp, 577 bp) and C. parapsilosis (520 bp) respectively. NC: Negative Control. L: 100 bp DNA size marker. 
decades. Several studies have evaluated the incidence of candidiasis, particularly in patients with hematologic malignancies. The most abundant oral fungal infection is candidiasis. In the oral tissues, candidiasis may become visible as pseudomembranous white plaques, erythematous areas, chronic atrophic white plaques, or angular cheilitis $(15,16)$. Immunosuppression patients are at high risk of life-threatening infection diseases. These patients are more susceptible to systemic infection, particularly those who need chemotherapy for hematologic malignancies (17). Immunocompetent patients scarcely present with oral candidiasis (18). In recent decades, candidiasis has been recognized as a infections affecting in immunosuppression patients with hematologic malignancies $(19,20)$.

Our study showed the prevalence of oral candidiasis among patients with leukemias and lymphomas was estimated to be $28 \%$. Similar results were reported in the present study by Schelenz et al. (20.5\%), Aghadavoudi et al. (29.2\%), Lone et al. (22\%), and Maheronnaghsh et al. (19.5\%) (12, 20-22). Gonzalez et al. on the other hand reported a higher rate of oral candidiasis i.e. $69.35 \%$ in cancer patients (4). This result

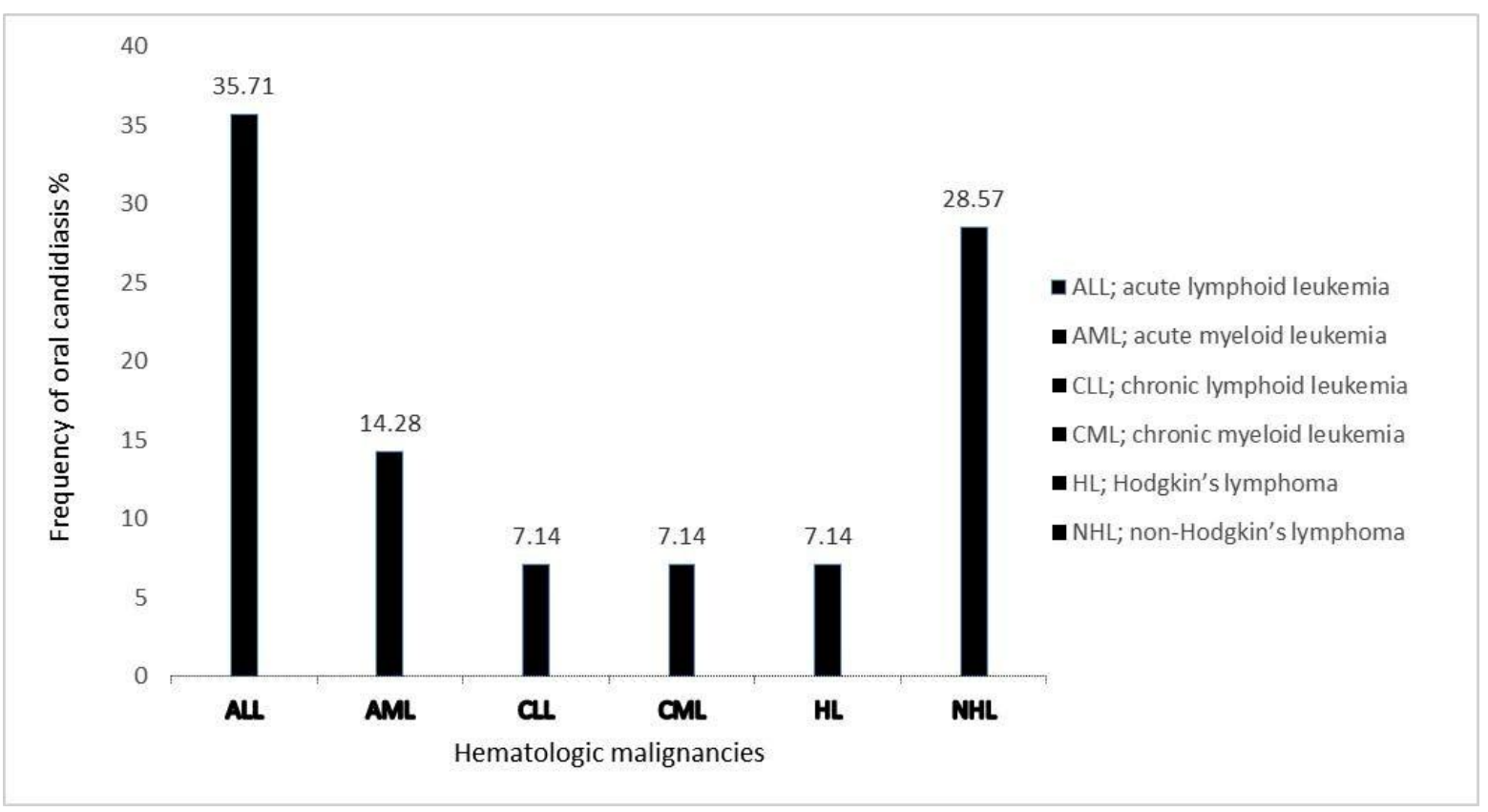

Fig. 3. Frequency of oral candidiasis in different types of hematologic malignancies.

Table 2. The frequency distribution of Candida species according to type of hematologic malignancies.

\begin{tabular}{llllllll}
\hline & \multicolumn{7}{c}{ Type of malignancies } \\
\cline { 2 - 8 } Candida spp & ALL & AML & CLL & CML & HL & NHL & Frequency (\%) \\
\hline C. albicans & 3 & 1 & 1 & 1 & 1 & 1 & 8 \\
C. glabrata & 0 & 0 & 0 & 0 & 0 & 2 & 2 \\
C. parapsilosis & 1 & 0 & 0 & 0 & 0 & 1 & 2 \\
C. krusei & 1 & 0 & 0 & 0 & 0 & 0 & 1 \\
C. kefyr & 0 & 1 & 0 & 0 & 0 & 0 & 1 \\
No Candida spp & 8 & 11 & 4 & 4 & 3 & 6 & 36 \\
Total & 13 & 13 & 5 & 5 & 4 & 10 & 50 \\
\hline
\end{tabular}

ALL, acute lymphoid leukemia; AML, acute myeloid leukemia; CLL, chronic lymphoid leukemia; CML, chronic myeloid leukemia; HL, Hodgkin's lymphoma; NHL, non-Hodgkin's lymphoma 
was contradictory with to the findings of our study. This difference in results may be due to possible causes such as climatic conditions, type of nutrition, age and type of treatment received $(21,22)$. In the present study, the highest prevalence was observed in patients with acute lymphoid leukemia (ALL) and non-Hodgkin's lymphoma (NHL) respectively. The reason for this increase may be the prevalence of these two diseases among children with a weakened immunity system than adults. Various studies have showed that $C$. albicans is the most prevalent candida species isolated from cancer patients (23-25). Oral candidiasis is a frequent complication in pediatric oncology with $C$. albicans is the important etiologic agent, however, there is an association with of other Candida species (4). Our data confirm that $C$. albicans was responsible for the majority of the oral candidiasis. Similar to our finding, Badiei et al. and Aghadavoudi et al. showed that the most common candida species in patients with hematologic malignancies is $C$. albicans $(23,24)$. Also our results are in agreement with Schelenz et al. (12), Lone et al. (26), Maheronnaghsh et al. (27) and Gonzalez et al. (4). Although $C$. albicans continues to be the most common species involved, Shokohi et al. reported other less common Candida species, such as $C$. orthopsilosis, C. infanticola and C. spencermartinsiae, may be present in clinical specimens obtained from cancer patients (28). Based on our research, there is a higher chance of having a systemic infection in patients with hematological malignancies that have Candida colonization (29). Systemic infection can spread to the different organs and increases the mortality.

Oral candidiasis represents a problem for immunosuppressed patients (30). The treatment of hematological malignancies relies heavily on cytotoxic chemotherapy that most often places patients at risk for fungal infections due to disruptions in mucosal barrier integrity and neutropenia (2). Colonizing Candida species probably present as reservoir for future systemic candidiasis. In the present study, 28\% of immunocompromised patients were colonized by Candida species. C. albicans was the most prevalent species in patients with leukemia and lymphoma. The information about distribution of candida species can be useful for the management of systemic candidiasis in immunocompromised patients, early diagnosis and empirical antifungal therapies (31).

\section{ACKNOWLEDGEMENTS}

This research was supported by the research council of Kerman University of Medical Sciences, Kerman, Iran.

\section{REFERENCES}

1. Sharifynia S, Falahati M, Akhlaghi L, Foroumadi A, Fateh R. Molecular identification and antifungal susceptibility profile of Candida species isolated from patients with vulvovaginitis in Tehran, Iran. J Res Med Sci 2017; 22: 132.

2. Franco MP, Green JS, Young J-AH. Evaluation and management of bacterial and fungal infections in patients with a hematological malignancy: A 2018 update. Neoplastic Diseases of the Blood 2018: 1063-1078.

3. Guo F, Yang Y, Kang Y, Zang B, Cui W, Qin B, et al. Invasive candidiasis in intensive care units in China: a multicentre prospective observational study. J Antimicrob Chemother 2013; 68: 1660-1668.

4. Gonzalez Gravina H, Gonzalez de Moran E, Zambrano O, Lozano Chourio M, Rodriguez de Valero S, Robertis S, et al. Oral Candidiasis in children and adolescents with cancer: Identification of Candida spp. Med Oral Patol Oral Cir Bucal 2007; 12: E419-423.

5. Hajjeh RA, Sofair AN, Harrison LH, Lyon GM, Arthington-Skaggs BA, Mirza SA, et al. Incidence of bloodstream infections due to Candida species and in vitro susceptibilities of isolates collected from 1998 to 2000 in a population-based active surveillance program. $J$ Clin Microbiol 2004; 42: 1519-1527.

6. Marr KA, Carter RA, Crippa F, Wald A, Corey L. Epidemiology and outcome of mould infections in hematopoietic stem cell transplant recipients. Clin Infect Dis 2002; 34: 909-917.

7. Marr KA, Seidel K, White TC, Bowden RA. Candidemia in allogeneic blood and marrow transplant recipients: evolution of risk factors after the adoption of prophylactic fluconazole. J Infect Dis 2000; 181: 309-316.

8. Martino R, Lopez R, Sureda A, Brunet S, Domingo-Albos A. Risk of reactivation of a recent invasive fungal infection in patients with hematological malignancies undergoing further intensive chemo-radiotherapy. A single-center experience and review of the literature. Haematologica 1997; 82: 297-304.

9. Muhlemann K, Wenger C, Zenhausern R, Tauber MG. Risk factors for invasive aspergillosis in neutropenic patients with hematologic malignancies. Leukemia 2005; 19: 545-550.

10. Nucci M, Spector N, Bueno AP, Solza C, Perecmanis T, 
Bacha PC, et al. Risk factors and attributable mortality associated with superinfections in neutropenic patients with cancer. Clin Infect Dis 1997; 24: 575-579.

11. Pagano L, Girmenia C, Mele L, Ricci P, Tosti ME, Nosari A, et al. Infections caused by filamentous fungi in patients with hematologic malignancies. A report of 391 cases by GIMEMA infection program. Haematologica 2001; 86: 862-870.

12. Schelenz S, Abdallah S, Gray G, Stubbings H, Gow I, Baker P, et al. Epidemiology of oral yeast colonization and infection in patients with hematological malignancies, head neck and solid tumors. J Oral Pathol Med 2011; 40: 83-89.

13. Badiee P, Kordbacheh P, Alborzi A, Zakernia M, Haddadi P. Early detection of systemic candidiasis in the whole blood of patients with hematologic malignancies. Jpn J Infect Dis 2009; 62: 1-5.

14. Mirhendi H, Makimura K, Khoramizadeh M, Yamaguchi H. A one-enzyme PCR-RFLP assay for identification of six medically important Candida species. Nihon Ishinkin Gakkai Zasshi 2006; 47: 225-229.

15. Chen Y-K, Hou H-A, Chow J-M, Chen Y-C, Hsueh P-R, Tien H-F. The impact of oral herpes simplex virus infection and candidiasis on chemotherapy-induced oral mucositis among patients with hematological malignancies. Eur J Clin Microbiol Infect Dis 2011; 30: 753-759.

16. Epstein JB, Beier Jensen S. Management of hyposalivation and xerostomia: criteria for treatment strategies. Compend Contin Educ Dent 2015; 36: 600-603.

17. Shimada Y, Nakagawa Y, Ide K, Sato I, Hagiwara S, Yamada $\mathrm{H}$, et al. Importance of eliminating potential dental focal infection before the first cycle of chemotherapy in patients with hematologic malignancy. Support Care Cancer 2017; 25: 1379-1381.

18. Telles DR, Karki N, Marshall MW. Oral fungal infections: diagnosis and management. Dent Clin North Am 2017; 61: 319-349.

19. Haron E, Feld R, Tuffnell P, Patterson B, Hasselback R, Matlow A. Hepatic candidiasis: an increasing problem in immunocompromised patients. Am J Med 1987; 83: 17-26.

20. Thaler M, Pastakia B, Shawker TH, Oleary T, Pizzo PA. Hepatic candidiasis in cancer patients: the evolving picture of the syndrome. Ann Intern Med 1988; 108 : 88-100.

21. Shokri H, Khosravi AR, Yalfani R. Antifungal effica- cy of propolis against fluconazole-resistant Candida glabrata isolates obtained from women with recurrent vulvovaginal candidiasis. Int J Gynaecol Obstet 2011; 114: $158-159$.

22. Aghadavoudi F, Chabavizadeh J, Dehghan P, Moafi A. The frequency of oral candidiasis in children and adolescents with Leukemia in Isfahan province, Iran. JIMS 2017; 35: 151-156.

23. Dahiya MC, Redding SW, Dahiya RS, Eng TY, Kirkpatrick WR, Coco BJ, et al. Oropharyngeal candidiasis caused by non-albicans yeast in patients receiving external beam radiotherapy for head-and-neck cancer. Int J Radiat Oncol Biol Phys 2003; 57: 79-83.

24. Kumar CPG, Sundararajan T, Menon T, Venkatadesikalu M. Candidosis in children with onco-hematological diseases in Chennai, south India. Jpn J Infect Dis 2005; 58: 218-221.

25. Tekeli A, Dolapci I, Cesur S, Tekeli E, Icli F. [Candida dubliniensis studies and isolation of Candida types in oropharyngeal specimens from oncologic patients]. Mikrobiyol Bul 2002; 36: 57-63.

26. Bashir H, Ahmad J. Oral Candida colonization and infection in cancer patients and their antifungal susceptibility in a tertiary care hospital. Int J Adv Res 2014; 2: 541-550.

27. Maheronnaghsh M, Tolouei S, Dehghan P, Chadeganipour M, Yazdi M. Identification of Candida species in patients with oral lesion undergoing chemotherapy along with minimum inhibitory concentration to fluconazole. Adv Biomed Res 2016; 5: 132.

28. Shokohi T, Aslani N, Ahangarkani F, Meyabadi MF, Hagen F, Meis JF, et al. Candida infanticola and Candida spencermartinsiae yeasts: Possible emerging species in cancer patients. Microb Pathog 2018; 115: 353-357.

29. Marodi L, Johnston Jr RB. Invasive Candida species disease in infants and children: occurrence, risk factors, management, and innate host defense mechanisms. Curr Opin Pediatr 2007; 19: 693-697.

30. de Morales Rojas T, Zambrano O, Rivera L, Navas R, Chaparro N, Bernardonni C, et al. Oral-disease prevention in children with cancer: testing preventive protocol effectiveness. Med Oral 2001; 6: 326-334.

31. Sepulveda E, Brethauer U, Morales R, Jimenez M. Oral manifestations in pediatric patients wíth oncologic pathologic. Med Oral 2000; 5: 193-197. 